\title{
APROVECHAMIENTO DE LOS RECURSOS NATURALES EN LA COMUNIDAD EL PEÑóN, TEMASCALTEPEC: UNA RUTA ECOTURÍSTICA PARA LA DIVERSIFICACIÓN DE LA OFERTA
}

\author{
OMAR ISMAEL RAMÍREZ HERNÁNDEZ \\ omar.ramirez.hdez@hotmail.com \\ ROCÍO JARAMILLO JARAMILLO \\ rociojaramillojaramillo00@gmail.com \\ Centro Universitario UAEM Temascaltepec, \\ Universidad Autónoma del Estado de México
}

El uso de los recursos naturales ha constituido una nueva forma para los residentes de obtener ingresos; sin embargo, estos deben utilizarse de forma responsable. Por ello, el objetivo de este trabajo es generar una propuesta de ruta ecoturística en la comunidad de El Peñón, Temascaltepec, Estado de México, con el propósito de diversificar la oferta existente. A partir de un enfoque cualitativo, se desarrolló un estudio documental y descriptivo, dividido en dos etapas: la primera, contextualizó el destino e identificó la percepción de los residentes hacia proyectos turísticos; la segunda, realizó un ejercicio de planificación. Los resultados destacan que la comunidad percibe de buena manera al turismo y les gustaría participar en proyectos; asimismo, resaltan tres recursos naturales sobre los que basar la ruta: El Peñón del Diablo, la cascada y el lago.

Palabras clave: ecoturismo, ruta turística, diversificación, oferta, Temascaltepec.

\section{MANAGEMENT OF NATURAL RESOURCES IN THE COMMUNITY OF El PEÑón, TEMASCALTEPEC: AN ECOTOURISM ROUTE FOR THE DIVERSIFICATION OF THE OFFER}

The use of natural resources has become a new way for residents to obtain incomes; however, these must be used responsibly. Therefore, the objective of this work is to generate a proposal for an ecotourism route in the community of El Peñón, Temascaltepec, State of Mexico, with the purpose of diversifying the existing offer. Based on a qualitative approach, a documentary and descriptive study was developed, divided into two stages: the first contextualized the destination and identified the residents' perception towards tourism projects; while the second carried out a planning exercise. The results highlight that the community has a good perception of tourism and would like to participate in projects; they also remark three natural resources on which the route is based:The Devil's Rock, the waterfall and the lake.

Keywords: ecotourism, tourist route, diversification, offer, Temascaltepec.

Fecha de recepción: 9 de junio de 2020 Fecha de aceptación: 11 de septiembre de 2020.

CÓMO CITAR: Ramírez, O. y Jaramillo, R. (2020). Aprovechamiento de los recursos naturales en la comunidad El Peñón, Temascaltepec: una ruta ecoturística para la diversificación de la oferta. Dimensiones Turísticas, 4(7), 125-150. https://doi.org/10.47557/CTID4910 


\section{Introducción}

$\mathrm{H}$

oy en día, el turismo se ha convertido en una importante actividad a nivel mundial, principalmente por los beneficios económicos que implica. Sin embargo, se reconoce que también ha generado impactos negativos en los destinos, en su mayoría en el aspecto medioambiental (Camacho-Ruíz et al., 2016). Por ello, y como una manera de combatir los costos, surge el ecoturismo, el cual tiene por objetivo salvaguardar los recursos naturales y detonar el desarrollo sustentable de los sitios (Agaton-Lorenzo y Cruz-Vicente, 2013; Bringas-Rábago y Ojeda-Revah, 2000; Camacho-Ruíz et al., 2016; Obombo y Velarde, 2018; Orgaz y Cañero, 2015).

El ecoturismo es presentado como una opción para la diversificación de la oferta turística, con el aprovechamiento del entorno natural (Agaton-Lorenzo y Cruz-Vicente, 2013; Bringas-Rábago y Ojeda-Revah, 2000; Pinkus-Rendón y Pinkus-Rendón, 2015). En ese sentido, la finalidad de este trabajo es generar una propuesta de ruta ecoturística en la localidad de El Peñón, ubicada en el municipio de Temascaltepec, Estado de México. Cabe señalar que el lugar de estudio cuenta con una gran riqueza ambiental, la cual genera una incipiente pero constante afluencia de visitantes provenientes de Valle de Bravo, uno de los destinos consolidados del Estado de México.

Se elaboró este documento a fin de plasmar opciones tendientes a diversificar la oferta turística en la comunidad de El Peñón, centrada en la actualidad en eventos deportivos de ala delta y parapente. Adicionalmente, dicha propuesta se justifica en que existe una necesidad de formular proyectos de este tipo, donde los residentes tengan otras opciones de generar ingresos, al realizar actividades relacionadas con el turismo (Ramírez et al., 2020).

Para alcanzar el objetivo antes planteado, se diseñó una investigación documental y descriptiva que recupera la propuesta de Arredondo et al. (2013) y Ramírez (2019), quienes delinean una ruta y un proyecto turístico, respectivamente. La metodología es de enfoque cualitativo, aplicada en dos etapas: la primera, denominada preliminar, donde se contextualizó la comunidad en cuestión, al retomar fuentes secundarias e información proporcionada por los habitantes, y la segunda, denominada de planificación en la que se realizó el inventario y la propuesta de ruta ecoturística. Dicho esfuerzo es una oportunidad para hacer que los residentes valoren los recursos naturales, sensibilizándolos para su conservación y aprovechamiento sostenible. 


\section{Ecoturismo y las rutas turísticas}

En los últimos años, el ecoturismo se ha posicionado en el interés académico. De acuerdo con Weaver y Lawton (2007), diversos investigadores han realizado aportes a la comprensión del ecoturismo, motivados por la problemática del turismo de masas, el cual ha originado múltiples impactos en los aspectos sociales, económicos y medioambientales (Camacho-Ruíz et al., 2016; Wall y Mathieson, 2006). En este último aspecto, su premisa retoma fuerza ante la necesidad del uso responsable de los recursos naturales para la actividad turística.

De acuerdo con la bibliografía especializada, Ceballos Lascuráin es señalado como el padre del ecoturismo, al ser uno de los primeros en brindar una definición al respecto:

Es aquella modalidad turística ambientalmente responsable, consistente en viajar a o visitar áreas naturales relativamente sin disturbar, con el fin de disfrutar, apreciar y estudiar los atractivos naturales (paisaje, flora y fauna silvestres) de dichas áreas, así como cualquier manifestación cultural (del presente y del pasado) que pueda encontrarse ahí, a través de un proceso que promueve la conservación, tiene bajo impacto negativo ambiental y cultural, y propicia un involucramiento activo y socioeconómicamente benéfico de las poblaciones locales. (Ceballos, 1989, pp. 13-14).

De tal manera, el ecoturismo guarda estrecha relación con las actividades realizadas en la naturaleza, sin modificar los recursos o el medio ambiente donde se desarrollan. Si bien la definición de Ceballos propone una visión general del tema, de acuerdo con Fennell (2001), a principios del siglo XX existían más de 85 conceptos de ecoturismo, de los cuales se recuperan a continuación los más relevantes (ver tabla 1). 


\section{Tabla 1. Conceptos de ecoturismo}

\begin{tabular}{|l|l|}
\hline \multicolumn{1}{|c|}{ Autor y año } & \multicolumn{1}{c|}{ Definición } \\
\hline Lanquar (2001, p. 135) & $\begin{array}{l}\text { "Puede ser definido como un turismo de participación con la naturaleza, que se } \\
\text { diferencia de las demás modalidades (turismo rural y agroturismo)". }\end{array}$ \\
\hline $\begin{array}{l}\text { Organización Mundial del } \\
\text { Turismo }(2002, \text { p. 16) }\end{array}$ & $\begin{array}{l}\text { "Turismo en espacios poco actuados por el hombre, que debe contribuir a la } \\
\text { protección de la naturaleza y al bienestar de las poblaciones locales". }\end{array}$ \\
\hline Secretaría de Turismo (2004, p. 3) & $\begin{array}{l}\text { "Los viajes que tienen como fin el realizar actividades recreativas de apreciación y } \\
\text { conocimiento de la naturaleza, a través del contacto con la misma". }\end{array}$ \\
\hline Vanegas $(2006$, p. 1) & $\begin{array}{l}\text { "Es una actividad que combina la pasión por el viaje y lleva de la mano la } \\
\text { preocupación que se tiene por el medio ambiente". }\end{array}$ \\
\hline Jalani $(2012$, citado en Orgaz y & $\begin{array}{l}\text { "Aquel tipo de turismo que se desarrolla en áreas naturales y tiene como objetivo la } \\
\text { conservación de las zonas naturales y el desarrollo de las comunidades locales". }\end{array}$ \\
\hline Choral, s. f., párr. 23) & $\begin{array}{l}\text { "Se basa en el trabajo de la naturaleza, incluso en las áreas más sensibles y } \\
\text { restrictivas para el acceso humano y, al mismo tiempo, la preocupación por la } \\
\text { conservación/protección del medio ambiente". }\end{array}$ \\
\hline
\end{tabular}

Fuente: Elaboración propia con base en los autores citados.

Así, la tabla 1 retoma conceptos del ecoturismo, los cuales coinciden en el uso responsable de los recursos naturales para el aprovechamiento turístico. Las diferencias que presentan se deben a que fueron generadas para abordar contextos específicos, como las distintas actividades que se llevan a cabo bajo esta modalidad (Pinkus-Rendón y Pinkus-Rendón, 2015).

Algunos autores consideran que el ecoturismo se ha convertido en una actividad masificada (Bringas-Rábago y Ojeda-Revah, 2000; Mat y Baum, 2005; Pinkus-Rendón y Pinkus-Rendón, 2015); sin embargo, y desde un punto de vista optimista, otros investigadores postulan que el ecoturismo es visto como la actividad de mayor potencial en el sector para detonar el progreso local y regional, además de salvaguardar el medio ambiente (Ghorbani et al., 2015; Orgaz y Cañero, 2015). En particular, Ibarra-Michel y Velarde (2016) argumentan que favorece en el aspecto económico, ecológico y social de las regiones donde se lleva a cabo.

Por lo tanto, también es vinculado con la gestión comunitaria y el desarrollo sustentable, ante la necesidad de formular una planificación turística que involucre a los actores relacionados y las localidades (Ramírez, 2019; Suárez et al., 2013), lo cual ayuda a la generación de beneficios y la mitigación de impactos negativos, principalmente en el ámbito natural y social. Autores como Garduño y Guzmán (2012) y Ghorbani et al. (2015) comparten esta postura. 
Desde la perspectiva de aprovechar correctamente los elementos naturales, a fin de crear productos turísticos, la Secretaría de Turismo (Sectur, 2004) enlista aquellas actividades a realizar como parte del ecoturismo: talleres de educación ambiental, observación geológica, de ecosistemas y sideral, contemplación de fauna y fauna, safari fotográfico, avistamiento de fenómenos y atractivos especiales de la naturaleza, senderismo interpretativo, caminata recreativa, participación en programas de rescate de la vegetación y los animales, observación de fósiles y participación en proyectos de investigación biológica.

Por su parte, Drumm et al. (2004) proponen que un proyecto ecoturístico debe tener seis elementos básicos:

1) Bajo impacto natural en el área donde es llevado a cabo.

2) Involucrar en las fases de planeación, desarrollo, implementación y monitoreo a todos los actores relacionados directa o indirectamente.

3) Respetar las culturas y tradiciones locales.

4) Producir ingresos sostenibles y equitativos para todos los actores participantes.

5) Generar ingresos para la conservación de los recursos naturales utilizados.

6) Sensibilizar a los actores sobre su rol en la conservación de los recursos naturales.

Retomando lo anterior, es factible elaborar productos relacionados con el ecoturismo, tal es el caso de las rutas turísticas, que son identificadas como una de las actividades más sencillas y redituables, debido a que se requiere una inversión mínima, utilizando los recursos existentes (Arredondo et al., 2013; Baum, 1998; Bicudo et al., 2017). En este tenor, Hernández (2011) considera que su origen se encuentra en los recorridos históricos, realizados en lugares donde acontecieron hechos importantes que cuentan con un valor social distintivo.

Aunque existen diferentes definiciones sobre las rutas turísticas (Ibarra-Michel y Velarde, 2016; Obregón et al., 2016), de manera general son referidas como los productos en los cuales se realiza un traslado de un punto geográfico a otro, combinando tanto elementos naturales como culturales, donde los turistas son orientados sobre los lugares y duración del trayecto.

En este tenor, se distinguen diferentes tipos (Alpízar y Maldonado, 2009; Arredondo et al., 2013; Barragán, 2017; Jeambey, 2016; Kraftchick et al., 2014), que surgen del espacio geográfico y los recursos que predominan en el espacio de acción (tabla 2). 
Tabla 2. Tipos de rutas turísticas

\begin{tabular}{|l|l|}
\multicolumn{1}{|c|}{ Tipo } & \multicolumn{1}{c}{ Recursos utilizados } \\
\hline Cultural & Recursos culturales intangibles y tangibles, monumentos, museos. \\
\hline Gastronómica & Alimentos típicos o distintivos. \\
\hline Del vino & Plantíos de vid, instalaciones vitivinícolas. \\
\hline Cervecera & Fábricas, comercializadoras de cerveza, bares y restaurantes especializados. \\
\hline Ecoturística & Recursos naturales. \\
\hline
\end{tabular}

Fuente: Elaboración propia con base en la literatura revisada.

De esta forma, la ruta cultural está basada precisamente en los elementos culturales tangibles e intangibles (Arredondo et al., 2013); la gastronómica emplea la elaboración y degustación de alimentos típicos o distintivos de un lugar o región (Jeambey, 2016); la del vino realiza recorridos en haciendas productoras de esta bebida (Alpízar y Maldonado, 2009); la cervecera se especializa en la cata y procesos de elaboración de esta bebida (Kraftchick et al., 2014); y la ecoturística se enfoca en el aprovechamiento sustentable de los recursos naturales (Barragán, 2017). Cabe destacar que estos tipos de ninguna manera constituyen la totalidad, pues actualmente es posible encontrar otros elementos que pueden fungir como atractivos para organizar recorridos.

\section{Diversificación de la oferta a partir de la elaboración de rutas turísticas}

Distintos autores reconocen que la generación de productos novedosos ayuda a la captación de turistas, al incrementar la derrama económica y el tiempo de estadía de los visitantes (García y Doumet, 2017; Luján et al., 2016; Urquiola y Lalangui, 2016). Asimismo, existen trabajos que realizan la propuesta de actividades ecoturísticas para la diversificación de la oferta; tal es el caso de Agaton-Lorenzo y Cruz-Vicente (2013), quienes evidencian que los recorridos ecoturísticos en la Laguna de Tres Palos, Acapulco, aportan variantes a un destino conocido por el turismo de sol y playa.

En el mismo sentido, García y Doumet (2017) diseñan un producto de turismo basado en la gestión comunitaria en el cantón Bolívar, Ecuador, en donde estructuran dos rutas con base en actividades relacionadas al entorno natural de seis comunidades. Adicionalmente, los autores proponen las estrategias de comercialización y promoción de dichas rutas, con el fin de aprovechar los recursos existentes y contribuir al desarrollo de la región. 
De igual forma, Luján et al. (2016), en su intención de lograr un manejo integral costero de las comunidades, identifican alternativas que promueven la diversificación de la economía en la localidad de Pehuen-Co, provincia de Buenos Aires, Argentina. Con la zonificación del área de estudio, se determinó su grado de avance turístico y se detectaron nuevos sitios con potencial, los cuales podrían aprovecharse a través de recorridos.

Gonzaga et al. (2015) proponen una ruta ecoturística a través de la gestión comunitaria en la provincia de Zamora Chinchipe, Ecuador. Al plantear este proyecto, los autores buscan mejorar la calidad de vida de los residentes, con el objeto de generar mayores oportunidades económicas que fomenten su participación.

Visto desde un enfoque empresarial, las rutas diversifican los productos en un destino (Urquiola y Lalangui, 2016), pues aprovechan los recursos para establecer actividades atractivas y frescas (Garduño y Guzmán, 2012; Gonzaga et al., 2015). Esta nueva oferta igualmente trata de mitigar problemáticas sociales, económicas y medioambientales en los diferentes sitios. Por ello, resulta de vital importancia planear adecuadamente las rutas ecoturísticas, con el fin de alcanzar el desarrollo sustentable (Pinkus-Rendón y Pinkus-Rendón, 2015).

\section{Metodología para la generación de rutas turísticas}

La elaboración de productos turísticos no tiene una metodología aceptada en lo general; existen diversas posturas que consideran las necesidades y características de las regiones, algunas de ellas son elaboradas por el sector público, privado o académico. En este sentido, Ramírez (2019) realiza una revisión de diferentes formas de estructurar dichos productos presentando 15 metodologías. El autor expone la poca participación de la comunidad en cada una de ellas, por lo que sugiere que los habitantes en conjunto sean el pilar fundamental en la creación y gestión, situación que es apoyada por Reyes et al. (2017), quienes argumentan que en muchas ocasiones los residentes se limitan a prestar servicios o incorporarse a empresas foráneas, situación que complica alcanzar una mejora local.

En la abundancia de metodologías sin un consenso al respecto, García y Doumet (2017) exponen su propuesta, dividida en tres grandes fases: a) diagnóstico, b) estudio del mercado, c) diseño y comercialización. Este proceso es usado específicamente para la gestión comunitaria en el cantón Bolívar, Ecuador. 
Arredondo et al. (2013) realizan otro planteamiento enfocado en crear rutas turísticas que den valor al patrimonio cultural en el sur del estado de Jalisco. Su documento contiene 11 pasos a seguir, en donde los autores analizan factores de accesibilidad, apertura, oferta turística, patrimonio cultural, actores relacionados y la disponibilidad temporal del turista. La aplicación de la metodología incluye sugerencias para la comercialización y posicionamiento de la ruta como producto turístico cultural en la región.

Por su parte, Gonzaga et al. (2015) proponen que para este fin son necesarias tres etapas: la primera, dirigida a la generación de un intercambio entre actores y autoridades locales, al establecer un camino de cooperación mutua; la segunda, centrada en realizar un prediagnóstico del lugar, con un inventario de recursos para su aprovechamiento turístico; por último, la tercera etapa que consiste en la evaluación técnica y la propuesta de actividades a realizar en el área.

De esta forma, la planeación de rutas y en general de los productos turísticos, es un trabajo heterogéneo, el cual consiste en la búsqueda de beneficios económicos, medioambientales y sociales (Machado y Hernández, 2007); asimismo, el éxito de cada uno depende de diversos factores, como los recursos, la inversión, los objetivos, la gestión, las políticas públicas y la participación de la comunidad local (Hernández, 2011; Ibarra-Michel y Velarde, 2016; Ramírez, 2019).

Por lo hasta aquí argumentado, se decide proponer un nuevo producto turístico con el fin de diversificar la oferta del destino en cuestión, orientado a la generación de otras actividades de turismo, lo cual conllevará a la creación de empleo en la zona, a la par del aprovechamiento y la conservación de los recursos naturales, con elementos de participación de la población local, pues de acuerdo con Ramírez (2019) este factor es fundamental para el éxito de los proyectos turísticos.

\section{Proceso metodológico}

Para cumplir con el objetivo antes planteado de diseñar una propuesta de ruta ecoturística y atendiendo las propuestas de Arredondo et al. (2013) y Ramírez (2019), se estableció un planteamiento para el caso de la localidad, basado en sus características sociodemográficas y turísticas. De esta forma, la metodología fue establecida como un proceso dividido en dos etapas: a) preliminar y b) de planificación (figura 1). 
En preparación para la segunda etapa de la ruta ecoturística, se realizó en primer lugar un trabajo documental a través de la búsqueda de información sobre el área, seguido de un análisis descriptivo donde se identificaron las características generales de los recursos, las percepciones de la comunidad local hacia el turismo y la propuesta de ruta.

Para llevar a cabo el proyecto se utilizó un enfoque cualitativo y valiéndose de la experiencia de uno de los autores del presente texto, quien reside en la zona, fue posible contactar informantes y obtener datos relevantes sobre la comunidad y su dinámica turística. En contraparte, otro de los autores no estaba familiarizado con el contexto local, lo que contribuyó a incrementar la objetividad del estudio. A continuación, se describe cada etapa con los pasos, técnicas e instrumentos utilizados para el trabajo de campo.

Figura 1. Proceso metodológico

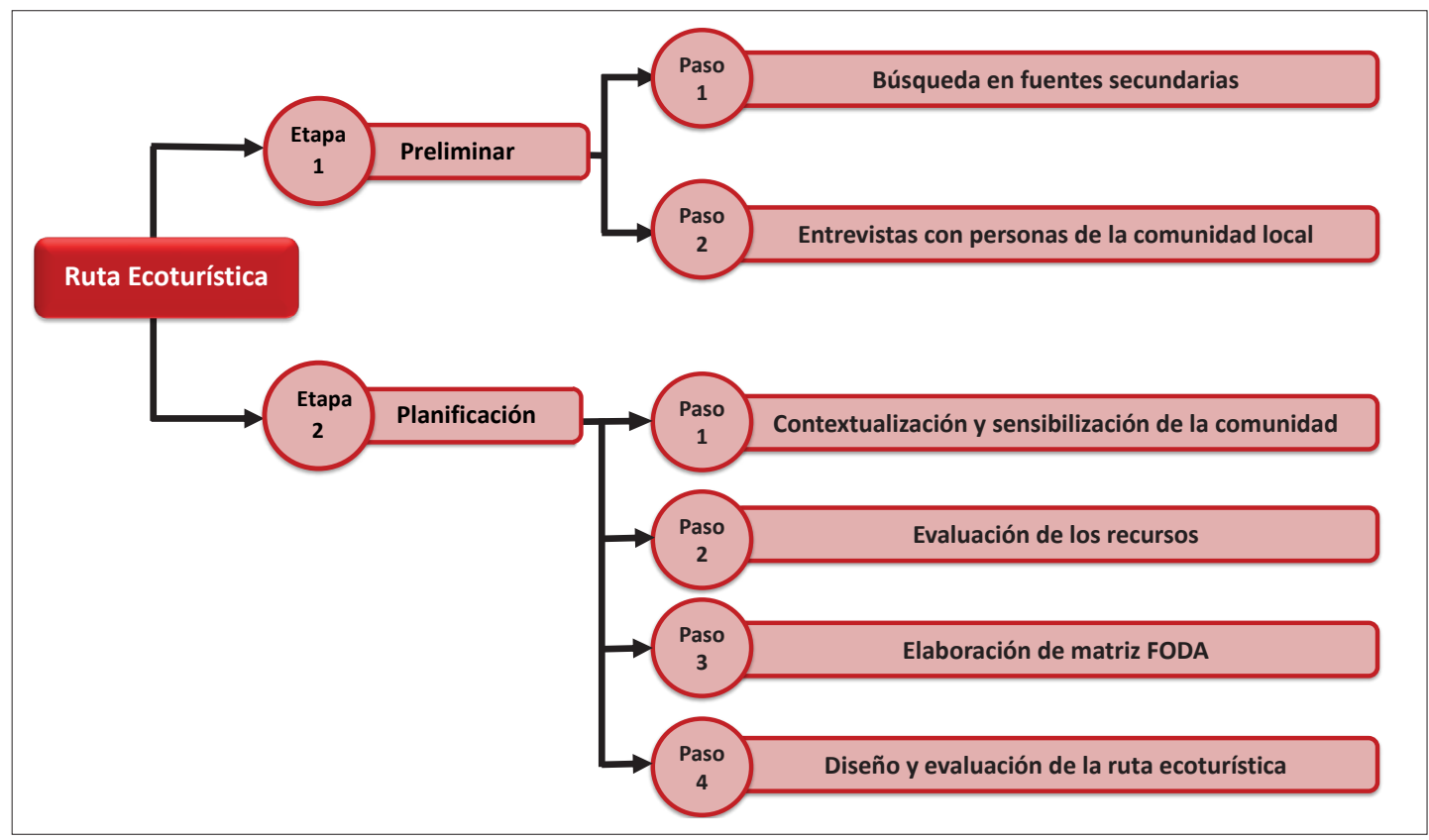

Fuente: Elaboración propia.

\subsection{Etapa preliminar}

Esta fase consistió en la búsqueda de información sobre la comunidad de El Peñón, dividida en dos pasos: 
Paso 1. Fuentes secundarias. Se indagó en documentos tales como planes de desarrollo municipal, artículos científicos, páginas web y la observación de campo en la propia comunidad.

Paso 2. Fuentes primarias. Radicó en preguntar directamente a los habitantes de la comunidad El Peñón sobre aspectos propios de la localidad; para ello, fue elaborado un guion de entrevista semiestructurada, el cual es un instrumento técnico para la obtención de información mediante la interacción verbal entre dos personas (entrevistador y entrevistado) (Bryman, 2012). Dicho documento contenía 12 preguntas, directamente las tres primeras ahondaban respecto a elementos sociodemográficos, las restantes versaban sobre la dinámica turística y social en el lugar, la postura hacia el turismo y nuevos proyectos turísticos, así como la evaluación de los recursos naturales y culturales existentes.

El muestreo utilizado fue por saturación teórica, el cual consiste en detener la actividad cuando las personas repiten los mismos datos y no proporcionan detalles nuevos (Bryman, 2012). La aplicación de las entrevistas se realizó durante los meses de octubre y diciembre de 2018, periodo en el que se buscó obtener respuestas de residentes de la comunidad de El Peñón con diferentes perfiles. Así, de 35 posibles participantes fueron 30 las personas entrevistadas, ya que cinco de ellas se negaron a colaborar en el estudio (tabla 3).

Tabla 3. Perfil de los informantes

\begin{tabular}{|c|c|c|c|c|c|}
\hline Variable & Categoría & Número & Variable & Categoría & Número \\
\hline \multirow{2}{*}{ Sexo } & Femenino & 10 & \multirow{4}{*}{ Edad } & 18 a 30 años & 12 \\
\hline & Masculino & 20 & & 31 a 45 años & 5 \\
\hline \multirow{6}{*}{ Ocupación } & Agricultor & 9 & & 46 a 60 años & 7 \\
\hline & Ama de casa & 8 & & 60 o más años & 6 \\
\hline & Comerciante & 5 & \multirow{4}{*}{ Nivel de estudios } & Primaria & 6 \\
\hline & Arquitecto & 1 & & Secundaria & 8 \\
\hline & Fotógrafo & 1 & & Preparatoria & 7 \\
\hline & Estudiante & 6 & & Universidad & 9 \\
\hline
\end{tabular}

Nota: Por motivos de confidencialidad y anonimato, los nombres de los entrevistados fueron modificados. Fuente: Elaboración propia con base en la investigación de campo. 
De esta manera, el grupo de informantes se integró con 10 hombres y 20 mujeres, de entre 18 y 78 años. Dentro de sus ocupaciones se ubican las de ama de casa (nueve) y agricultor (ocho), le siguen estudiante (seis) y comerciante (cinco). Las menos referidas son las de arquitecto (uno) y fotógrafo (uno).

Cabe resaltar que 22 individuos mencionaron dedicarse ocasionalmente a actividades turísticas durante la temporada de vuelo (noviembre a febrero), en la cual se desempeñan como choferes (cinco), vendedoras de alimentos y bebidas (seis) y ayudantes generales (11). Asimismo, es importante resaltar que todos los entrevistados han vivido siempre en el lugar de estudio.

\subsection{Etapa de planificación}

Con el análisis de la información recabada en la primera etapa, se prosiguió a la planificación de la ruta ecoturística, por medio de cuatro pasos:

Paso 1. Contextualización y percepción de la comunidad. Se caracterizó la comunidad de El Peñón, además se analizó la información obtenida en las entrevistas individuales con integrantes de la localidad, sobre la percepción de la actividad turística.

Paso 2. Evaluación de recursos. Se valoraron aquellos recursos susceptibles de aprovechamiento ecoturístico mediante fichas de inventario. Para ello, fue adaptada la propuesta elaborada por el Ministerio de Comercio, Industria y Turismo de Colombia (Mincit, 2010), con puntos como: datos del recurso, descripción, actividades desempeñadas, vías de acceso, estado de conservación, señalética, seguridad, equipamiento y fotografías; asimismo, fueron incorporados aspectos como actividades turísticas potenciales a realizar, tiempo para llegar al recurso y si recibe actualmente visitantes.

Igualmente, fue retomada información de la primera etapa, donde se cuestionó a las personas de la comunidad si los recursos eran susceptibles de uso turístico. Los factores que influyeron en la elección fueron el reconocimiento e importancia para los residentes, la accesibilidad, y que los locales aceptaran utilizar dichos elementos para el destino. Así, se determinó cuáles eran aprovechables para la actividad ecoturística.

Paso 3. Elaboración de matriz de Fortalezas, Oportunidades, Debilidades y Amenazas (FODA). Se realizó un análisis FODA en torno a la comunidad de El Peñón, Temascaltepec, considerando los datos recabados durante la etapa 1 y el trabajo de campo. 
Paso 4. Diseño y evaluación de la ruta ecoturística. Derivado de los pasos previos, se procedió a confeccionar la ruta, con fundamento en los recursos aprovechables desde el punto de vista de la comunidad local, además de establecer los posibles servicios y actividades a ofertar. De igual manera, se realizó una propuesta de actores (residentes y patrocinadores potenciales) susceptibles de identificar su pertinencia.

\section{Resultados}

Los resultados obtenidos a raíz de la metodología planteada se dividen en cuatro apartados: el primero está relacionado con la etapa preliminar (contextualización y percepción de la comunidad El Peñón, Temascaltepec), los otros tres fueron generados en la etapa de planificación (evaluación de recursos, matriz FODA, diseño y evaluación de la ruta ecoturística).

\subsection{Contextualización y percepción de la comunidad El Peñón, Temascaltepec}

Temascaltepec es un municipio del sur del Estado de México, colinda al noreste con Zinacantepec, al noroeste con Valle de Bravo, al norte con Amanalco, al oeste con Zacazonapan, al sur con San Simón de Guerrero y Texcaltitlán, al sureste con Coatepec Harinas y al suroeste con Tejupilco ( $\mathrm{H}$. Ayuntamiento de Temascaltepec, 2016) (figura 2). De acuerdo con la Gaceta Municipal, la entidad tiene una superficie total de $546.79 \mathrm{~km}^{2}$ y su población total es de 35 mil 904 habitantes.

Los climas predominantes a lo largo del municipio son el subhúmedo (54\%), el Iluvioso tropical (28\%), el semifrío subhúmedo (14\%) y el cálido subhúmedo (2\%) (H. Ayuntamiento de Temascaltepec, 2016). Asimismo, sin contar la cabecera municipal, el lugar es considerado como zona rural. En cuanto a su economía, Temascaltepec desarrolla la agricultura, la ganadería y la floricultura. Adicionalmente, es reconocida su histórica producción minera; en la actualidad, la compañía First Majestic Silver Corp. tiene concesionada la mina llamada La Guitarra, en la localidad de La Albarrada, donde existen yacimientos de vetas y mantos de sulfuros masivos (Subsecretaría de Minería, 2018).

De igual manera, el turismo ha tratado de posicionarse como una actividad relevante en el lugar, el cual desde el año 2009 ostenta el nombramiento de Pueblo con encan- 
to, reconocimiento otorgado por la Secretaría de Turismo Estatal debido a sus atractivos y recursos con vocación turística (H. Ayuntamiento de Temascaltepec, 2016).

Figura 2. Ubicación de Temascaltepec

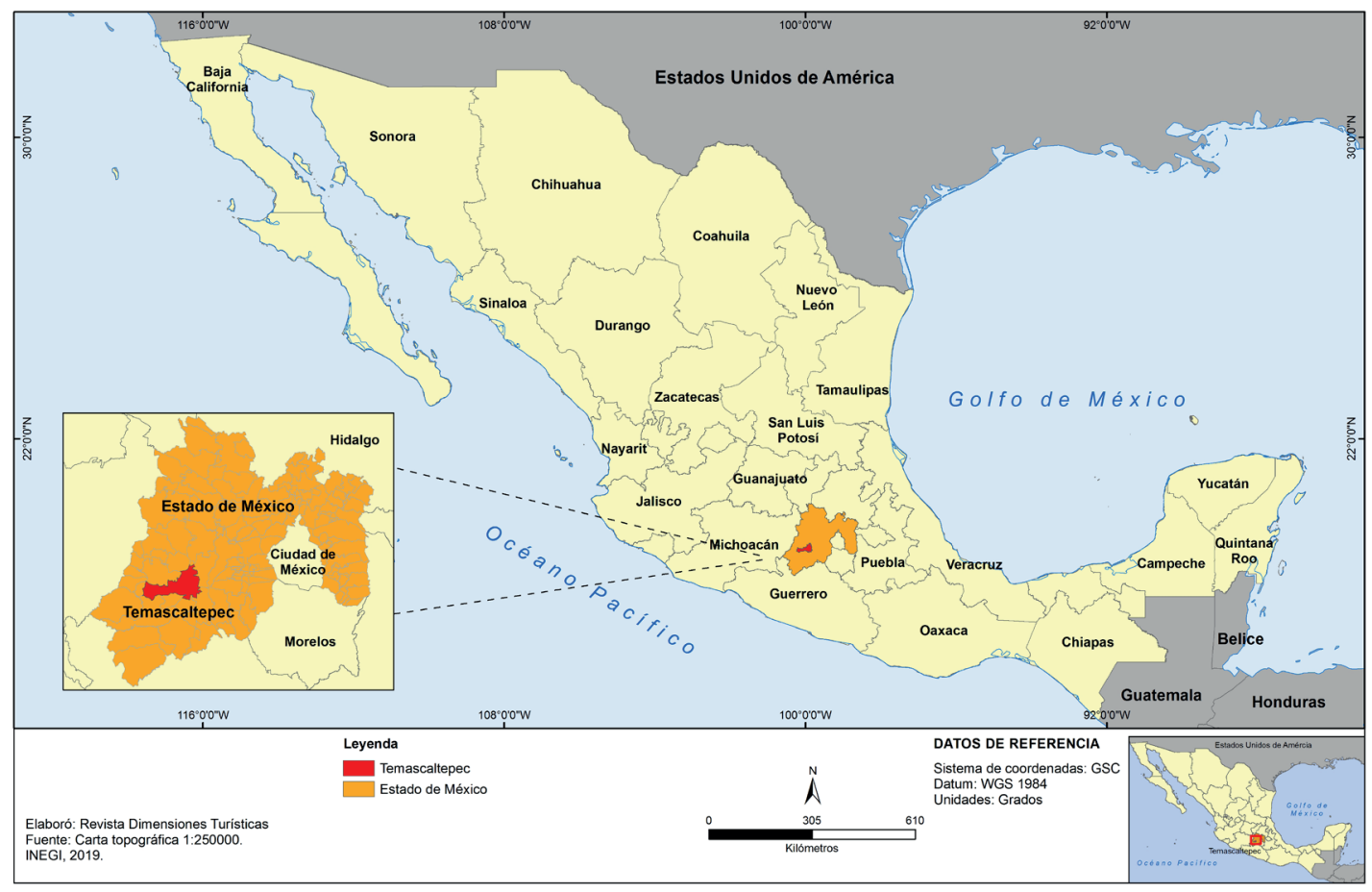

Fuente: Ver interior figura 2.

La comunidad de El Peñón se localiza en el municipio de Temascaltepec. De acuerdo con datos de la Secretaría de Desarrollo Social (Sedesol, 2015), el lugar cuenta con 533 habitantes, de los cuales 274 son hombres y 259 mujeres. Al igual que el municipio al que pertenece, las principales actividades en la localidad son la agricultura y ganadería de traspatio, ya que no se cuenta con actividades industriales o de manufactura.

Es de considerar que el nivel de marginación social y de rezago es alto (Sedesol, 2015). Durante las entrevistas, y por experiencia del autor que reside en la zona, no fue notoria una fuerte migración a Estados Unidos como en épocas pasadas, pero las personas han dejado la comunidad para ir a ciudades cercanas como Toluca y la Ciudad de México. 
En el ámbito turístico, en El Peñón son llevadas a cabo diferentes actividades, entre las que destacan el vuelo en ala delta y parapente, deportes por los que es conocida la comunidad a nivel internacional. De igual manera, existe la práctica de rappel, ciclismo y campismo, aunque de forma incipiente. Aunado a esto, la infraestructura turística es limitada, pues en la actualidad solo existen dos hoteles que brindan sus servicios únicamente de noviembre a febrero.

Si bien la localidad representa un punto de interés para el sector turismo, no se tienen datos oficiales sobre el número de visitantes que arriban. Sin embargo, los informantes mencionan que en temporada alta o de vuelo (noviembre a febrero) llegan alrededor de 50 a 60 turistas a la semana, mientras que en temporada baja (marzo a octubre) los visitantes llegan hasta 25. Además, los locales observan que los turistas son jóvenes con gusto por las actividades que posibilitan el contacto con la naturaleza y la práctica deportiva.

Adicionalmente, la mayoría de los entrevistados (24) coincide en que los turistas que arriban lo hacen procedentes de Valle de Bravo, el destino más reconocido de la región, e indican que muchos de los visitantes solo están un periodo de tiempo corto en la comunidad de El Peñón. En este sentido, Joaquín, comerciante, comenta que:

La generación de nuevos productos turísticos en la comunidad ayudaría a que los visitantes no solo vengan a volar (parapente o ala delta) y se regresen a Valle, que es donde ellos hacen todo lo demás (actividades turísticas). (Comunicación personal).

Por lo anterior, de acuerdo con las entrevistas se infiere la razón principal de que 23 de los 30 informantes consideran relevante crear nuevos proyectos turísticos en la comunidad, lo cual coincide con otras investigaciones (García y Doumet, 2017; Garduño y Guzmán, 2012; Gonzaga et al., 2015), donde los residentes son capaces de visualizar el beneficio económico de este tipo de actividad. A la par, algunos de los participantes mencionan que esto combatiría la estacionalidad de visitantes, tal como lo dice Ximena, ama de casa:

Algunos de los que nos dedicamos en nuestros tiempos libres al turismo, o como una actividad complementaria, solo vemos más ingresos cuando es temporada de vuelo, pues en otra época son pocos los que vienen y de ellos casi nadie se queda. (Comunicación personal). 
Existen algunos ejemplos donde la nueva oferta turística ayuda a cambiar la estacionalidad de la demanda en los destinos (Alpízar y Maldonado, 2009; Bicudo et al., 2017; Calçada, 2017); no obstante, esos lugares ya tienen una afluencia importante de turistas, por lo que no podría esperarse un comportamiento similar en la comunidad de El Peñón, debido a sus características. Sin embargo, sí podrían integrarse servicios novedosos para los turistas que llegan y detonar de esta manera una derrama económica adicional a la presente.

Por su parte, los entrevistados identifican que la actividad turística trae otros beneficios, como la enseñanza de los deportes practicados por los turistas. En este sentido, Jesús, fotógrafo de profesión, señala que:

La escuela de vuelo que está en la comunidad enseña a los chavos a volar de forma gratuita (parapente), les proporciona todo, el equipo y los instructores; clases que son muy caras... más o menos 30 mil pesos o a veces hasta más. (Comunicación personal).

Por lo anterior, se asume la responsabilidad social de las empresas turísticas en la comunidad, uno de los puntos que también ayuda a detonar el desarrollo en el lugar, con bienestar para los residentes (Pinkus-Rendón y Pinkus-Rendón, 2015; Soares et al., 2015).

\subsection{Evaluación de recursos}

En las entrevistas realizadas a los integrantes de la comunidad El Peñón, ellos reconocen ocho recursos, de los cuales tres son naturales y cinco culturales (tabla 4). 


\section{Tabla 4. Recursos de la comunidad El Peñón}

\begin{tabular}{|c|c|c|c|}
\hline Recurso & Descripción & Evaluación & $\begin{array}{l}\text { Posibles actividades } \\
\text { por realizar }\end{array}$ \\
\hline $\begin{array}{l}\text { Canales y } \\
\text { subestación } \\
\text { eléctrica }\end{array}$ & $\begin{array}{l}\text { Es una extensión cercana a los } 600 \mathrm{~m} \\
\text { de longitud, que en un inicio fue creada } \\
\text { para la generación de electricidad en } \\
\text { la comunidad, pertenece al régimen de } \\
\text { propiedad comunal. }\end{array}$ & $\begin{array}{l}\text { No aprovechable: debido } \\
\text { a que actualmente se } \\
\text { encuentra abandonada y } \\
\text { no existe infraestructura, ni } \\
\text { señalamientos en el lugar. }\end{array}$ & Paseos a pie o en bicicleta. \\
\hline La capilla & $\begin{array}{l}\text { Iglesia católica construida } \\
\text { aproximadamente hace } 50 \text { años, donde } \\
\text { son celebrados eventos religiosos en } \\
\text { torno a San Antonio de Padua y misas } \\
\text { dominicales. }\end{array}$ & $\begin{array}{l}\text { No aprovechable: a pesar de } \\
\text { encontrarse en buen estado, } \\
\text { no cuenta con el interés de los } \\
\text { visitantes. }\end{array}$ & $\begin{array}{l}\text { Asistencia a fiesta patronal } \\
\text { en el mes de junio. }\end{array}$ \\
\hline Pan artesanal & $\begin{array}{l}\text { Es un alimento elaborado } \\
\text { artesanalmente, el cual puede } \\
\text { adquirirse en dos establecimientos de } \\
\text { la comunidad, el más reconocido es el } \\
\text { llamado "lasloyo". }\end{array}$ & $\begin{array}{l}\text { Aprovechable: cuenta con } \\
\text { potencial latente; sin embargo, } \\
\text { al ser un recurso cultural, no } \\
\text { tendría lugar protagónico en } \\
\text { la ruta. }\end{array}$ & $\begin{array}{l}\text { Degustación durante los } \\
\text { recorridos. }\end{array}$ \\
\hline Deshilados & $\begin{array}{l}\text { Es una fina artesanía creada con cortes } \\
\text { en la tela, su elaboración data de hace } \\
\text { más de } 60 \text { años en la comunidad. } \\
\text { Aún existen talleres donde enseñan a } \\
\text { realizarlos. }\end{array}$ & $\begin{array}{l}\text { Aprovechable: es un recurso } \\
\text { cultural, por lo que no sería } \\
\text { incluido en la ruta como una } \\
\text { actividad principal. }\end{array}$ & Souvenirs. \\
\hline La comunidad & $\begin{array}{l}\text { Es el espacio donde se encuentran } \\
\text { los habitantes; además, cuenta con } \\
\text { instalaciones que facilitan la reunión } \\
\text { de personas y es el lugar donde llega } \\
\text { el transporte público y particular con } \\
\text { visitantes. }\end{array}$ & $\begin{array}{l}\text { Aprovechable: no es un } \\
\text { recurso natural; sin embargo, } \\
\text { será incluido por sus } \\
\text { características, debido a las } \\
\text { posibles actividades a realizar. }\end{array}$ & $\begin{array}{l}\text { Talleres de educación } \\
\text { ambiental, de conservación } \\
\text { y aprovechamiento de } \\
\text { recursos. }\end{array}$ \\
\hline El Peñón & $\begin{array}{l}\text { El Peñón del Diablo es el nombre que le } \\
\text { da la comunidad a la formación rocosa } \\
\text { situada en el lugar; existe una gran } \\
\text { variedad de flora y fauna, entre la que } \\
\text { se puede encontrar árboles de encino y } \\
\text { ocote, así como ardillas, lagartijas, aves } \\
\text { e iguanas. }\end{array}$ & $\begin{array}{l}\text { Aprovechable: es el recurso } \\
\text { natural más importante de } \\
\text { la zona, cuenta con algunos } \\
\text { caminos no señalados para } \\
\text { llegar a él. }\end{array}$ & $\begin{array}{l}\text { Senderismo para llegar } \\
\text { hacia el recurso, rappel, } \\
\text { escalada, fotografía, } \\
\text { observación de fauna y } \\
\text { flora. }\end{array}$ \\
\hline La cascada & $\begin{array}{l}\text { Es una caída de agua de alrededor } \\
\text { de } 5 \mathrm{~m} \text { de alto, con un espacio de } 6 \mathrm{~m}^{2} \\
\text { donde se estanca el agua, con salida al } \\
\text { río. }\end{array}$ & $\begin{array}{l}\text { Aprovechable: es uno } \\
\text { de los recursos naturales } \\
\text { mencionados por la } \\
\text { comunidad y cuenta con } \\
\text { acceso en buen estado. }\end{array}$ & Actividades recreativas. \\
\hline El lago & $\begin{array}{l}\text { Es un cuerpo de agua con superficie } \\
\text { aproximada de } 45 \mathrm{~m}^{2} \text {. Su estado de } \\
\text { conservación es bueno y se encuentran } \\
\text { aves y peces. }\end{array}$ & $\begin{array}{l}\text { Aprovechable: a pesar de no } \\
\text { contar con señalamientos y } \\
\text { seguridad, son factibles las } \\
\text { actividades turísticas. }\end{array}$ & Pesca recreativa y kayak. \\
\hline
\end{tabular}

Fuente: Elaboración propia con base en el trabajo de campo. 
Dentro de los recursos naturales identificados, se destaca El Peñón como el de mayor atractivo. Pedro, agricultor, relata:

Lo más importante a visitar aquí es El Peñón, en primer lugar, porque así se llama la comunidad y, en segundo, por sus condiciones naturales, pues permite practicar diversas actividades, no solo en el sitio, sino en el camino tomado para llegar ahí. (Comunicación personal).

Adicionalmente, testimonios mencionan que la cascada y el lago tienen un gran valor comunitario, ya que en ellos los habitantes desarrollan actividades recreativas como la pesca y las caminatas. Llama la atención que los entrevistados subrayan con énfasis los paisajes naturales, los cuales, al igual que en el trabajo de Orgaz y Cañero (2015), podrían fungir como un atractivo cuando se realicen los recorridos.

Los informantes también señalaron cuatro recursos culturales; sin embargo, algunos de ellos explican que no resultan de interés para los turistas, tal es el caso de Lucía, ama de casa, quien comenta:

Los turistas visitan poco otros lugares aparte del despegue, el aterrizaje y El Peñón, pues ellos lo que quieren es practicar deporte o tener contacto con la naturaleza, lo que se puede hacer con toda tranquilidad aquí. (Comunicación personal).

Así, los elementos naturales utilizados para la propuesta serán El Peñón, la cascada, el lago y el centro de la comunidad; dejando a un lado los elementos culturales que, si bien son relevantes para los habitantes, en este caso fungirán como elementos complementarios de la ruta, pues al tener la temática ecoturística no es posible incorporarlos como principales.

\subsection{Matriz FODA}

A raíz de la información reunida en la revisión documental y el trabajo de campo, se marcaron las fortalezas, oportunidades, debilidades y amenazas de la comunidad El Peñón (tabla 5). 


\section{Tabla 5. Análisis FODA de El Peñón}

\section{Fortalezas}

Recibe afluencia de turistas en búsqueda de realizar actividades deportivas, de aventura y contacto con la naturaleza.

Cuenta con recursos naturales y culturales de importancia regional, que pueden ser aprovechados para la actividad turística.

Es reconocido el valor natural por los turistas que visitan el lugar.

Son celebradas competencias nacionales e internacionales en la comunidad.

Elaboración de productos de forma artesanal.

La población ve de forma positiva la actividad turística y le gustaría participar en proyectos.

Aprovechar los recursos naturales y culturales para implementar proyectos turísticos.

Existe el apoyo de las autoridades municipales para financiar proyectos productivos en la región.

Cercanía con el pueblo mágico de Valle de Bravo.

\section{Oportunidades}

Creciente interés de los turistas por desarrollar actividades en contacto con la naturaleza.

Incremento de actividades de turismo alternativo a nivel mundial.

La comunidad empieza a ser reconocida por los pilotos internacionales de parapente y ala delta.

Posibilidad para la diversificación de la economía local.

Existe la posibilidad de proponer políticas de conservación turística.

\section{Debilidades}

Los residentes tienen una escasa iniciativa emprendedora.

Solo cuenta con una escuela de vuelo en el lugar.

Ningún proyecto turístico está enfocado en el aprovechamiento adecuado de los recursos naturales.

Insuficiente infraestructura, señalización y servicios turísticos especializados.

No existe red de celular e Internet en la mayor parte de la localidad.

Insuficiente transporte público para llegar a la comunidad.

La población no está capacitada para recibir el turismo y no existen guías de turistas locales capacitados.

No existe una planificación para el desarrollo del turismo.

Casi todos los turistas que visitan la localidad lo hacen por empresas turísticas de Valle de Bravo.

Poca capacidad financiera de los residentes para invertir en proyectos turísticos.

\section{Amenazas}

Deterioro de recursos naturales debido a causas externas, como el cambio climático.

Imagen de inseguridad causada por el narcotráfico en la región.

Migración de la comunidad local hacia otros puntos geográficos.

Existen otros destinos consolidados en la región.

Incremento de la exigencia del turista.

Poco adiestramiento para afrontar factores relacionados con la salud, como epidemias o pandemias.

Crisis y recesión económica a nivel mundial.

Fuente: Elaboración propia con base en el trabajo de campo. 
De esta forma, es posible establecer la cercanía de la comunidad con el municipio de Valle de Bravo como una de las características que más influye en el análisis FODA, ya que la mayor cantidad de turistas provienen de ese sitio, lo que ocasiona una dependencia del mercado emisor. Esta situación podría beneficiar a la comunidad de El Peñón, como un referente en la planificación y gestión turística (Soares et al., 2015).

Una amenaza que podría influir en el desarrollo del destino y la ruta propuesta, es la imagen de inseguridad que mantiene la zona sur del Estado de México. Esta situación puede provocar que los turistas no arriben como se esperaría, toda vez que algunos autores destacan la seguridad del sitio turístico como una de las condicionantes que el visitante privilegia (Hernández y De la Torre, 2016; Martínez y Trejoluna, 2017).

\subsection{Diseño de la ruta ecoturística y propuesta de evaluación}

Con la información recolectada en la etapa preliminar y los pasos anteriores, se generó el diseño de la ruta ecoturística (figura 3), la cual contempla los recursos identificados con las características necesarias para ser aprovechados.

Figura 3. Diseño de ruta ecoturística

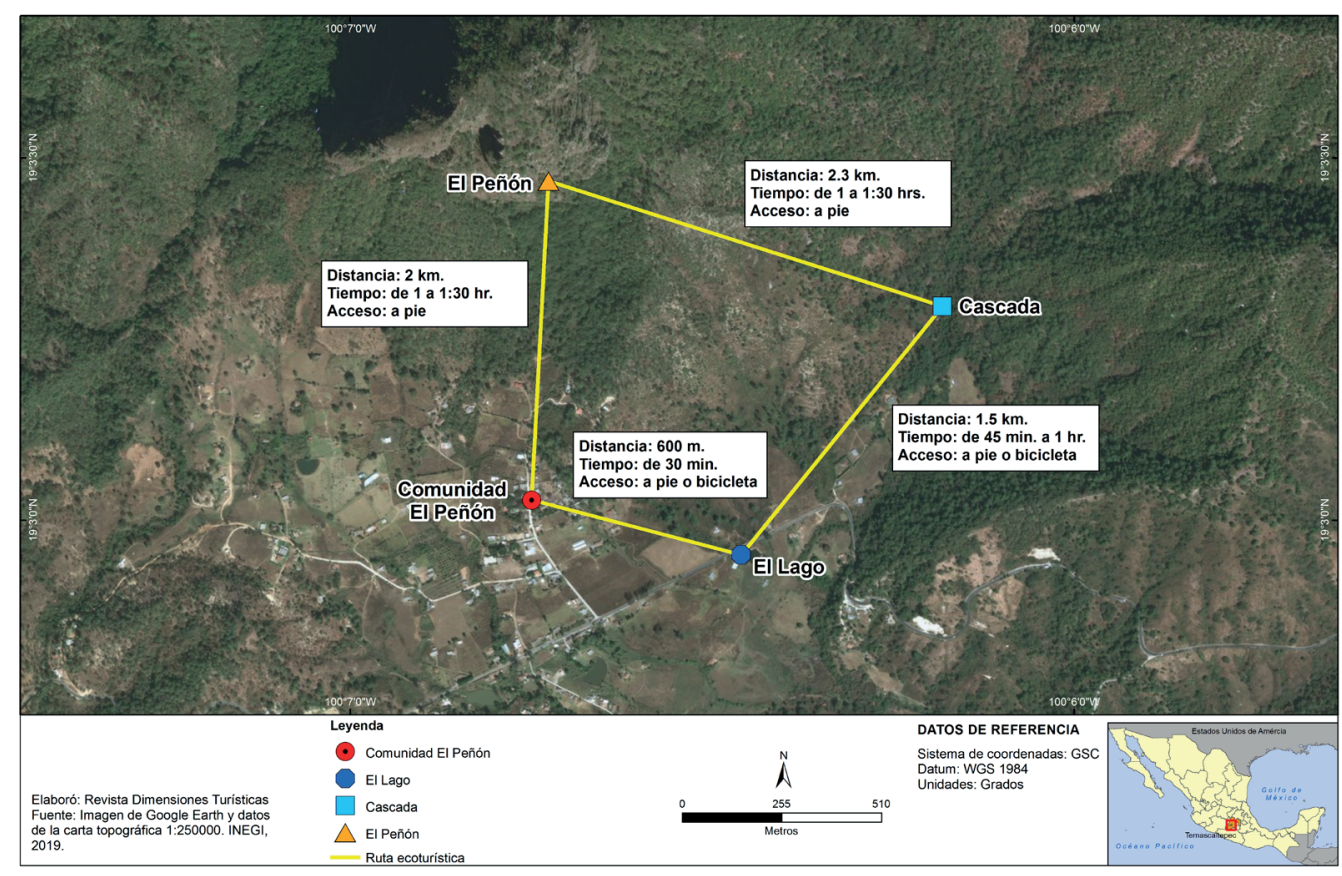

Fuente: Ver interior figura 3. 
De esta manera, la ruta daría comienzo en el centro de la comunidad, para después visitar el lago, la cascada y, por último, El Peñón; aunque también puede ser de forma inversa. Cabe mencionar que el acceso a los atractivos es posible a pie o en bicicleta, en donde se distingue que la distancia entre los puntos va de los $600 \mathrm{~m}$ hasta los $2.3 \mathrm{~km}$. El tiempo para llegar a los lugares es de 30 minutos a hora y media.

Al no contar con señalización de tránsito, es necesario que los turistas realicen el recorrido con integrantes de la comunidad local, pues son ellos quienes conocen el trayecto y los senderos específicos; así, los residentes se incorporarían a estas actividades. De acuerdo con Suárez et al. (2013), una de las primeras acciones para la inclusión de la comunidad en proyectos turísticos es hacerla partícipe en la operatividad, para que puedan darle seguimiento. Adicionalmente, una situación por atender es la profesionalización de los habitantes, ya que, según se reconoce en el trabajo de campo, solo algunos cuentan con las competencias necesarias para la atención al turista.

En cuanto a las actividades a realizar en la ruta establecida y su duración, estas dependerán del espacio donde se encuentren los turistas (tabla 6). Adicionalmente, los recursos culturales pueden aprovecharse cuando el visitante se encuentre en el centro de la comunidad El Peñón, pero no formarán parte del recorrido.

Tabla 6. Actividades y materiales dentro de la ruta

\begin{tabular}{|l|l|l|}
\hline \multicolumn{1}{|c|}{ Lugar } & \multicolumn{1}{|c|}{ Actividades } & \multicolumn{1}{c|}{ Materiales } \\
\hline El Peñón & $\begin{array}{l}\text { Observación de flora y fauna } \\
\text { Observación sideral }\end{array}$ & Binoculares \\
\hline Safari fotográfico & Telescopio astronómico \\
\hline Cascada & Actividades recreativas acuáticas & Cámara fotográfica \\
\hline Lago & Pesca recreativa & Traje de baño \\
\hline Ruta de tránsito & $\begin{array}{l}\text { Senderismo interpretativo y caminata } \\
\text { recreativa }\end{array}$ & Caña de pescar, anzuelos, carnada, red \\
\hline Comunidad El Peñón & $\begin{array}{l}\text { Talleres de educación ambiental y rescate } \\
\text { de flora y fauna }\end{array}$ & $\begin{array}{l}\text { Papelería (hojas, lápices, marcadores, } \\
\text { plumones), fotografías }\end{array}$ \\
\hline
\end{tabular}

Fuente: Elaboración propia.

De esta forma, las acciones son propuestas conforme a la literatura revisada, específicamente lo postulado por Sectur (2004), que ayudaría a generar un bajo impacto en la zona, premisa fundamental del ecoturismo. Además, a través del planteamiento se intenta producir ingresos sostenibles y equitativos para los participantes, así 
como la conservación de los recursos naturales, lo cual coincide con lo establecido por Drumm et al. (2004).

El proyecto de ruta ecoturística debe evaluarse por la comunidad local, instituciones públicas y posibles inversionistas/colaboradores, con el fin de que se ajusten elementos para la puesta en marcha de las acciones (Arredondo et al., 2013; Ramírez, 2019). De esta forma, durante el trabajo de campo fueron vislumbrados actores sociales, públicos y privados que pueden apoyar, entre los que destacan la Dirección de Turismo del Municipio de Temascaltepec, las escuelas de vuelo (Vuelo libre y Sky Riders), escuelas similares en Valle de Bravo, además de la comunidad local. Así, los elementos que se tendrían que evaluar son la pertinencia, la logística, las actividades a implementar $y$, de ser viable, realizar una prueba de mercado.

Adicionalmente, uno de los elementos que no cubre esta propuesta es identificar un camino a seguir, a fin de propiciar la participación de la comunidad y actores interesados en todas las fases de planificación, porque solo se centra en lo operativo y en la evaluación de recursos naturales. Atender este vacío es vital para el éxito de los productos turísticos, debido a que, como algunos autores evidencian (Garduño y Guzmán, 2012; Ghorbani et al., 2015; Ramírez, 2019), es importante conformar un grupo de trabajo que se haga cargo del proyecto y con ello fortalecer el trabajo comunitario.

\section{Conclusiones}

El ecoturismo se ha convertido en una práctica consolidada en los últimos años; principalmente debido a que promueve el desarrollo sustentable (Ghorbani et al., 2015; Orgaz y Cañero, 2015). De esta manera, los destinos han visto una oportunidad de innovar en su oferta, por medio del aprovechamiento de los recursos naturales (Agaton-Lorenzo y Cruz-Vicente, 2013; Calçada, 2017; Pinkus-Rendón y Pinkus-Rendón, 2015). Uno de los productos más utilizados para ello son las rutas turísticas (Ibarra-Michel y Velarde, 2016; Fernández y Guzmán, 2005), las cuales necesitan poca inversión y aprovechan los recursos poco utilizados para la recepción de turistas (Urquiola y Lalangui, 2016).

La contribución de este documento se observa a través de dos aspectos: a) la elaboración de una metodología para diseñar rutas ecoturísticas, y b) la propuesta de proyecto en la comunidad El Peñón, Temascaltepec, la cual busca diversificar la 
oferta en la temporada de vuelo. También se identificó que los residentes están de acuerdo en que se generen productos turísticos, lo cual para Ramírez (2019) es de vital importancia, si se pretende detonar el desarrollo sustentable.

En cuanto a las limitantes del estudio, la propuesta contempla solo en algunas etapas a la comunidad local, por lo que es indispensable trabajar con los residentes y los actores interesados a fin de llevarla a cabo. Además, una debilidad del proyecto es que no todos los habitantes están capacitados para la oferta de los servicios, además de que la infraestructura es limitada y se necesitaría un plan estratégico para su implementación.

Lo anterior constituye una oportunidad para futuras investigaciones, tal es el caso de la gestión comunitaria destinada a establecer el proyecto, planes de negocios, análisis de mercado, estudios del costo-beneficio y capacidad de carga, entre otros, que contribuyan a que se favorezca el desarrollo sustentable, local y turístico en la zona.

Por último, los resultados obtenidos ratifican que la ruta ecoturística puede diversificar la oferta de la comunidad de El Peñón, dando valor al aprovechamiento de los recursos naturales; sin embargo, y de acuerdo con Calderón-Puerta et al. (2018) es necesario plantear, desarrollar y evaluar la forma de gestión e implementación de los nuevos proyectos de turismo en las comunidades, con el fin de que los beneficios sean mayores a los costos generados por la actividad turística.

\section{Referencias}

Agaton-Lorenzo, D. y Cruz-Vicente, M. (2013). Propuesta de diversificación de la oferta turística en Acapulco: paseos ecoturísticos en la Laguna de Tres Palos. Turydes, Revista de Turismo y Desarrollo, 6(14). https://bit.ly/36NrVgl

Alpízar, V. y Maldonado, M. (2009). Integración de la ruta del vino en Querétaro, un producto innovador. Quivera, Revista de Estudios Territoriales, 11(2), 97-109. https://bit.ly/360JnBx

Arredondo, P., Hernández, C. y Mendoza, T. (2013). Propuesta para el diseño de rutas turísticas culturales: el caso del sur del estado de Jalisco, México. Turismo \& Sociedade, 6(2), 324-344. https://doi.org/10.5380/tes.v6i2.31931

Barragán, J. (2017). Diseño de una ruta ecoturística en el municipio de Coloso - Sucre: senderismo y biodiversidad [tesis de maestría, Universidad Tecnológica de Bolívar]. Biblioteca de la Universidad Tecnológica de Bolívar. https://bit.ly/2GHXqOB 
Baum, T. (1998). Taking the exit route: extending the tourism area life cycle model. Current Issues in Tourism, 1(2), 167-175. https://doi. org/10.1080/13683509808667837

Bicudo, R., Alves, M., Aparecida, S., Batistella, M. y Farinaci, J. (2017). Perspectives for environmental conservation and ecosystem services on coupled rural-urban systems. Perspectives in Ecology and Conservation, 15(2), 74-81. https://doi.org/10.1016/j.pecon.2017.05.005

Bringas-Rábago, N. L. y Ojeda-Revah, L. (2000). El ecoturismo: ¿una nueva modalidad del turismo de masas? Economía, Sociedad y Territorio, 2(7), 373-403. https://doi.org/10.22136/est002000436

Bryman, A. (2012). Social Research Methods. Oxford University Press. https://bit.ly/ 3nEXzCT

Calçada, F. (2017). La diversificación de la oferta turística como alternativa para minimizar la estacionalidad: el caso de los eventos en Balneário Camboriú (Santa Catarina, Brasil). Cuadernos de Turismo, 39, 91-112. https://bit.ly/2FifBcU

Calderón-Puerta, D. M., Arcila-Garrido, M. L. y López-Sánchez, J. A. (2018). Las rutas e itinerarios turístico-culturales en los portales oficiales de turismo de las comunidades autónomas españolas. Revista de Estudios Andaluces, 35, 123-145. https://doi.org/10.12795/rea.2018.i35.05

Camacho-Ruiz, E., Carrillo-Reyes, A., Rioja-Paradela, T. y Espinoza-Medinilla, E. (2016). Indicadores de sostenibilidad para el ecoturismo en México: estado actual. LiminaR, Estudios Sociales y Humanísticos, 14(1), 156-168. https://doi. org/10.29043/liminar.v14i1.429

Ceballos, H. (1989). The future of ecotourism. Mexico Journal, 1(17), 13-14.

Cheia, G. (2013). Ecotourism: definition and concepts. Revista de Turism: Studii si Cercetari in Turism, 15, 56-60. https://bit.ly/3ILHcmD

Drumm, A., Moore, A., Terborgh, J., Patterson, C. y Soles, A. (2004). Desarrollo del ecoturismo: un manual para los profesionales de la conservación (Vol. 2). Nature Conservancy United States. Agency for International Development. https:// bit.ly/2SWogF6

Fennell, D. (2001). A content analysis of ecotourism definitions. Current Issues in Tourism, 4(5), 403-421. https://doi.org/10.1080/13683500108667896

Fernández, G. y Guzmán, A. (2005). Patrimonio industrial y rutas turísticas culturales: algunas propuestas para Argentina. Cuadernos de Turismo, 15, 97-112. https://bit.ly/36XjQpA 
García, N. y Doumet, N. (2017). El producto turístico comunitario como estrategia para diversificar las economías locales del cantón Bolívar, provincia de Manabí, Ecuador. Revista Interamericana de Ambiente y Turismo, 13(1), 105-116. https://doi.org/10.4067/S0718-235X2017000100105

Garduño, M. y Guzmán, C. (2012). El turismo rural y la participación comunitaria en Ranchería Las Palomas, Estado de México. Revista Rosa Dos Ventos, 4(2), 235249. http://hdl.handle.net/20.500.11799/79539

Ghorbani, A., Raufirad, V., Rafiaani, P. y Azadi, H. (2015). Ecotourism sustainable development strategies using SWOT and QSPM model: a case study of Kaji Namakzar Wetland, South Khorasan Province, Iran. Tourism Management Perspectives, 16, 290-297. https://doi.org/10.1016/j.tmp.2015.09.005

Gonzaga, C., Moncayo, A., Cortés, S. y Suasnavas, G. (2015). Ruta ecoturística: una oportunidad para promover la acción comunitaria, base del desarrollo sustentable en la Amazonia Sur del Ecuador. Revista Cultura y Turismo, 67, 199227. https://bit.ly/3dftVzs

H. Ayuntamiento de Temascaltepec. (2016, 31 de marzo). Gobierno municipal de Temascaltepec 2016-2018. https://bit.ly/33ICwrl

Hernández, E. y De la Torre, M. (2016). Turismo y violencia. Los nuevos imaginarios del miedo. Opción, 32(13), 203-227. https://bit.ly/2GJD3QY

Hernández, J. (2011). Los caminos del patrimonio. Rutas turísticas e itinerarios culturales. PASOS, Revista de Turismo y Patrimonio Cultural, 9(2), 225-236. https:// doi.org/10.25145/j.pasos.2011.09.021

Ibarra-Michel, J. y Velarde, M. (2016). Rutas turísticas sustentables como alternativa para el desarrollo de comunidades rurales. En R. Espinoza, R. Chávez, y E. Andrade (Eds.), Estudios turísticos en regiones de México (pp. 179-209). Universidad de Guadalajara, Centro Universitario de la Costa.

Jeambey, Z. (2016). Rutas gastronómicas y desarrollo local: un ensayo de conceptualización en Cataluña. PASOS, Revista de turismo y patrimonio cultural, 14(5), 1187-1198. https://doi.org/10.25145/j.pasos.2016.14.079

Kraftchick, J. F., Byrd, E. T., Canziani, B. y Gladwell, N. J. (2014). Understanding beer tourist motivation. Tourism Management Perspectives, 12, 41-47. https://doi. org/10.1016/j.tmp.2014.07.001

Lanquar, R. (2001). Marketing turístico: de lo global a lo local. Ariel Turismo. 
Luján, M., Ferrelli, F. y Piccolo, M. (2016). Diversificación de la actividad turística de balnearios: el caso de Pehuen-Co, Argentina. Cuadernos de Turismo, 38, 245261. https://doi.org/10.6018/turismo.38.271441

Machado, E. y Hernández, Y. (2007). Procedimiento para el diseño de un producto turístico integrado en Cuba. Teoría y Praxis, 3(4), 161-174. https://doi. org/10.22403/uqroomx/typ04/10

Martínez, R. y Trejoluna, O. (2017). La percepción de seguridad de los turistas en un sitio de turismo religioso. International Journal of Scientific Management and Tourism, 3(4), 255-274. https://bit.ly/2SGHuys

Mat, A. y Baum, T. (2005). Mass-ecotourism: an alternative to sustainable tourism development in developing countries. TEAM, Journal of Hospitality \& Tourism, 2(1), 81-91. https://bit.ly/2SIMgLO

Ministerio de Comercio, Industria y Turismo (Mincit). (2010). Metodología para la elaboración del inventario de atractivos turísticos. https://bit.ly/3dl93GV

Obombo, K. y Velarde, M. (2018). Ecoturismo y conservación: Perspectivas y prácticas en las reservas de la biósfera de Los Tuxtlas, México y Maasai Mara, Kenia. Dimensiones Turísticas, 2(2), 53-78. https://doi.org/10.47557/PMQQ9256

Obregón, S. A., Sánchez, J. y Somohano, M. de L. (2016). Planificación de rutas turísticas para autobús a través de indicadores de accesibilidad integral y de dotación de bienes materiales e inmateriales. Transporte y Territorio, 14, 144166. https://bit.ly/3300GAU

Organización Mundial del Turismo (OMT). (2002). El mercado francés del ecoturismo. https://bit.ly/3nBldAo

Orgaz, F. y Cañero, P. (2015). El ecoturismo como motor de desarrollo en zonas rurales: un estudio de caso en República Dominicana. Ciencia y Sociedad, 40(1), 47-76. https://doi.org/10.22206/cys.2015.v40i1.pp47-76

Orgaz, F. y Moral, S. (s. f.). Las áreas protegidas como espacios para fomentar el desarrollo sostenible y el turismo comunitario. Un estudio de caso exitoso. Revista DELOS, Desarrollo Local Sostenible. https://bit.ly/3IsJ03F

Pinkus-Rendón, M. A. y Pinkus-Rendón, M.J. (2015). El ecoturismo: quimera o realidad de desarrollo en la reserva de la biosfera Ría Celestún, México. Revista LiminaR, Estudios Sociales y Humanísticos, 13(1), 69-80. https://doi.org/10.29043/ liminar.v13i1.367 
Ramírez, O. (2019). Propuesta metodológica para la generación de productos turísticos a partir de la comunidad local. RETOS, Revista de Ciencias de Administración y Economía, 9(17), 127-143. https://doi.org/10.17163/ret.n17.2019.08

Ramírez, O., Serrano, R. y Palmas, Y. (2020). Impactos socioculturales del turismo deportivo en la comunidad de El Peñón, Temascaltepec Methaodos, Revista de Ciencias Sociales, 8(1), 62-76. https://doi.org/10.17502/m.rcs.v8i1.338

Reyes, M., Ortega, Á. y Machado, E. (2017). Modelo para la gestión integrada del turismo comunitario en Ecuador, caso de estudio Pastaza. Revesco, Revista de Estudios Cooperativos, 123, 250-275. https://doi.org/10.5209/REVE.53242

Secretaría de Desarrollo Social (Sedesol). (2015). Catálogo de localidades. https://bit. ly/2GHkqNG

Secretaría de Turismo (Sectur). (2004). Turismo alternativo. Una nueva forma de hacer turismo (Fascículo 1). https://bit.ly/3jYBNIO

Soares, J., Ivars, J. y Gândara, J. (2015). La evolución de destinos turísticos litorales consolidados. Análisis comparado de Balneario Camnoriú (Brasil) y Benidorm (España). Anales de Geografía de la Universidad Complutense, 35(2), 143-166. https://doi.org/10.5209/rev_aguc.2015.v35.n2.50118

Suárez, R., Bribiescas, F. y Villanueva, J. (2013). Gestión y fortalecimiento de proyectos comunitarios: una opción para el desarrollo turístico sustentable en comunidades rurales del Estado de Chihuahua (Caso Ejido Ojo de la Casa). European Scientific Journal, 9(8), 270-283. https://bit.ly/3iOMNGT

Subsecretaría de Minería. (2018). Panorama minero del Estado de México. Secretaría de Economía. https://bit.ly/2laa2yd

Urquiola, O. y Lalangui, J. (2016). La ruta agro turística y de naturaleza Banagua de la provincia El Oro. Revista Universidad y Sociedad, 8(3), 128-134. https://bit. ly/3iMAMI8

Vanegas, G. (2006). Ecoturismo instrumento de desarrollo sostenible [monografía, Universidad de Antioquia]. Biblioteca Digital de la Universidad de Antioquía. https://bit.ly/3jNSaai

Wall, G. y Mathieson, A. (2006). Tourism: change, impacts and opportunities. Pearson - Prentice Hall.

Weaver, D. y Lawton, L. (2007). Twenty years on: the state of contemporary ecotourism research. Tourism Management, 28(5), 1168-1179. https://doi. org/10.1016/j.tourman.2007.03.004 